LALP $--84-27$

DE84 017107

\title{
The Delta: \\ The First Pion Nucleon Resonance Its Discovery and Applications
}

\author{
Darragh E. Nagle
}

\author{
DISCLAIMER
}

\begin{abstract}
This report was prepared as an account of work sponsored by an agency of the United States Government. Neither the United States Government nor any agency thereof, nor any of their employees, makes any warranty, express or implied, or assumes any legal liability or responsibility for the accuracy, completeness, or usefulness of any information, apparatus, product, or process disclosed, or represents that its use would not infringe privately owned rights. Reference herein to any specific commercial product, process, or service by trade name, trademark, manufacturer, or otherwise does not necessarily constitute or imply its endorsement, recommendation, or favoring by the United States Government or any agency thereof. The views and opinions of authors expressed herein do not necessarily state or reflect those of the United States Government or any agency thereof.
\end{abstract}

This article is based on a lecture given at the University of Chicago Symposium in honor of H. L. Anderson, May 11, 1982.
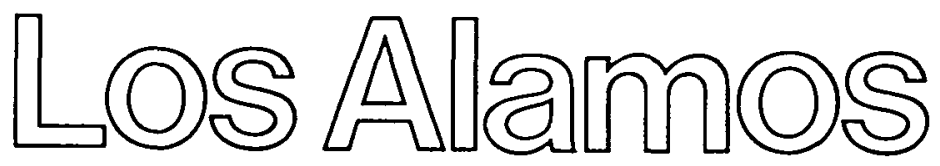

Los Alamos National Laboratory Los Alamos,New Mexico 87545 


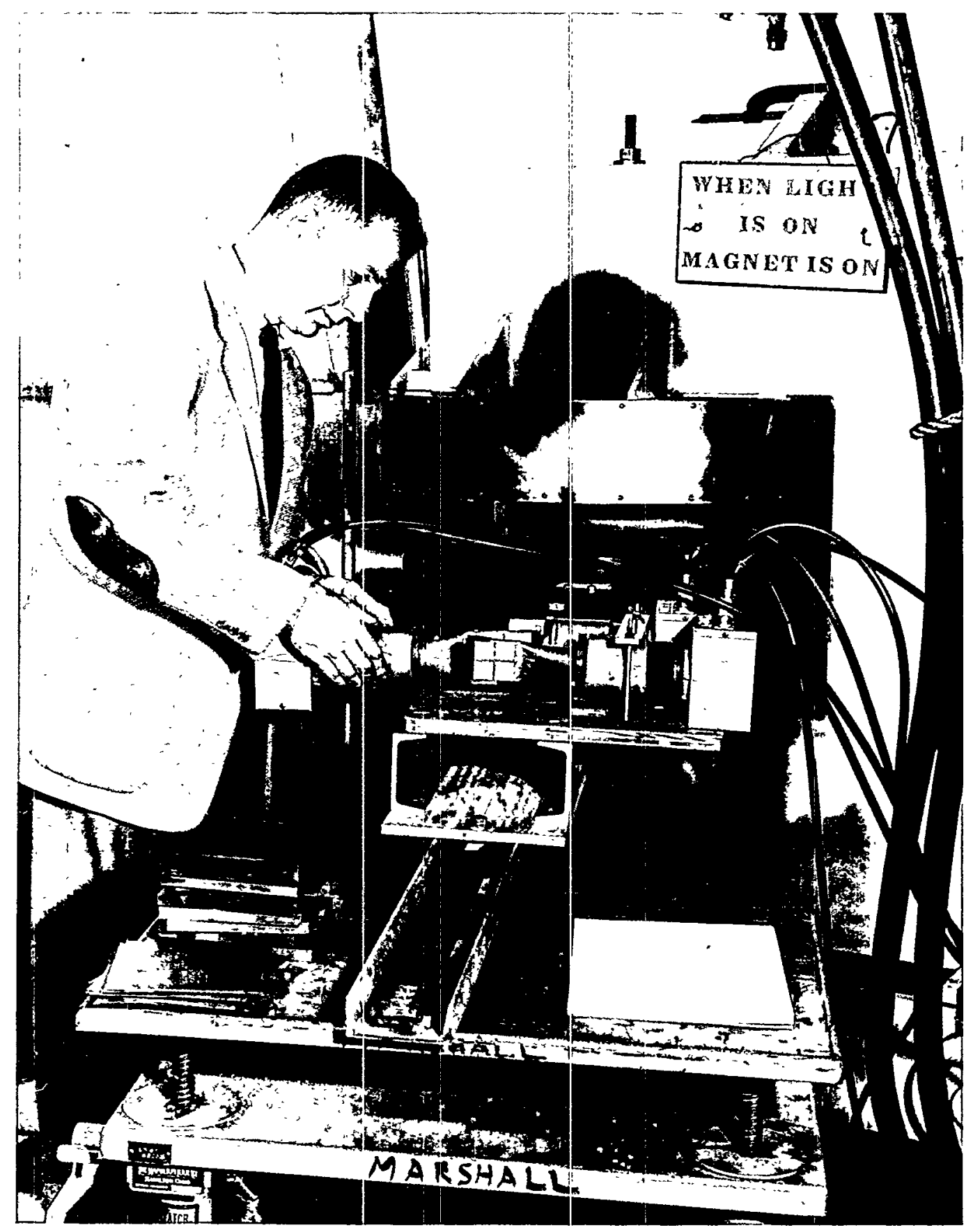

Herbert Anderson, adjusting some scintillation counters at the Chicago cyclotron, 1951. 


\section{DISCLAIMER}

Portions of this document may be illegible in electronic image products. Images are produced from the best available original document. 


\title{
THE DELTA: THE FIRST PION NUCLEON RESONANCE
}

\author{
Its Discovery and Applications
}

-Darragh E. Nagle

1

l'd like to try to recapture with you some of the fun and excitement of the pion-scattering work that led to the discovery of what is now called the delta particle. How significant this discovery was became apparent only gradually, as I will try to summarize for you. That the delta is alive today and thriving at Los Alamos (as well as other places) I shall also try to persuade you.

\section{Beginnings}

$\mathrm{T}$ he story begins for me back in 1940 at Columbia University where, as a shiny new graduate student, I thought I should pay a courtesy call on the illustrious Professor Enrico Fermi, whose presence at Columbia had been one reason for my coming. His office was listed as being on the seventh floor of Pupin Laboratory. So I took the elevator, got out at that floor, and looked around for the office. Suddenly a door burst open, and a dark-haired man ran past me at full tilt, disappearing through another door. He was wearing a lab coat and he was carrying a bit of something in a pair of chemical tongs. I stood there flat-footed wondering what to do, when the first door burst open again, and what appeared to be a younger version of the first gentleman, similarly attired and similarly burdened, dashed by me and also disappeared through the second door. Although the full significance of this minidrama was not completely clear to me then, ${ }^{*}$ it seemed clear enough that this was not the day for a courtesy call on Professor Fermi. Only later did I find myself caught up in the races devised by Fermi and

* The bit of something that was being carried was in fact a thin foil of rhodium metal, which had been activated by neutrons. Because rhodium decays with a half-life of 42 seconds, it was necessary to be quick in carrying it from the lab where it was activated to a place where its activity could be measured.
Herbert Anderson, at Chicago, then at Los Alamos, and then, in 1951, back at the University of Chicago again. The photo shows Anderson at the cyclotron in the 1950 s.

\section{Early Days at the Cyclotron}

$\mathrm{I}$ n 1951, the great Chicago cyclotron, constructed by Anderson following principles developed at Berkeley, was at the stage of final assembly and testing in the new building known as John Marshall's barn. The pictures, in order, show the cyclotron and the famous "trolley-car" target of Fermi, which ran along the periphery of the cyclotron magnet and in which the proton beam interacted to give pions, etc. A view from above shows the shield wall between the cyclotron and the experimental room. The slots in the steel were for several pion beams; they came at various "impact parameters" with respect to the cyclotron center, each slot corresponding to a different pion beam energy.

Because negative pions bend oppositely from the protons, the negative pions coming out were emitted in the forward direction. The positive pions were those emitted in the backward direction, and so the higher energy positive pion had lower intensity than the low-energy positive pion. In the initial operation, then, the positive pion energies were lower, a fact of some historical significance. 


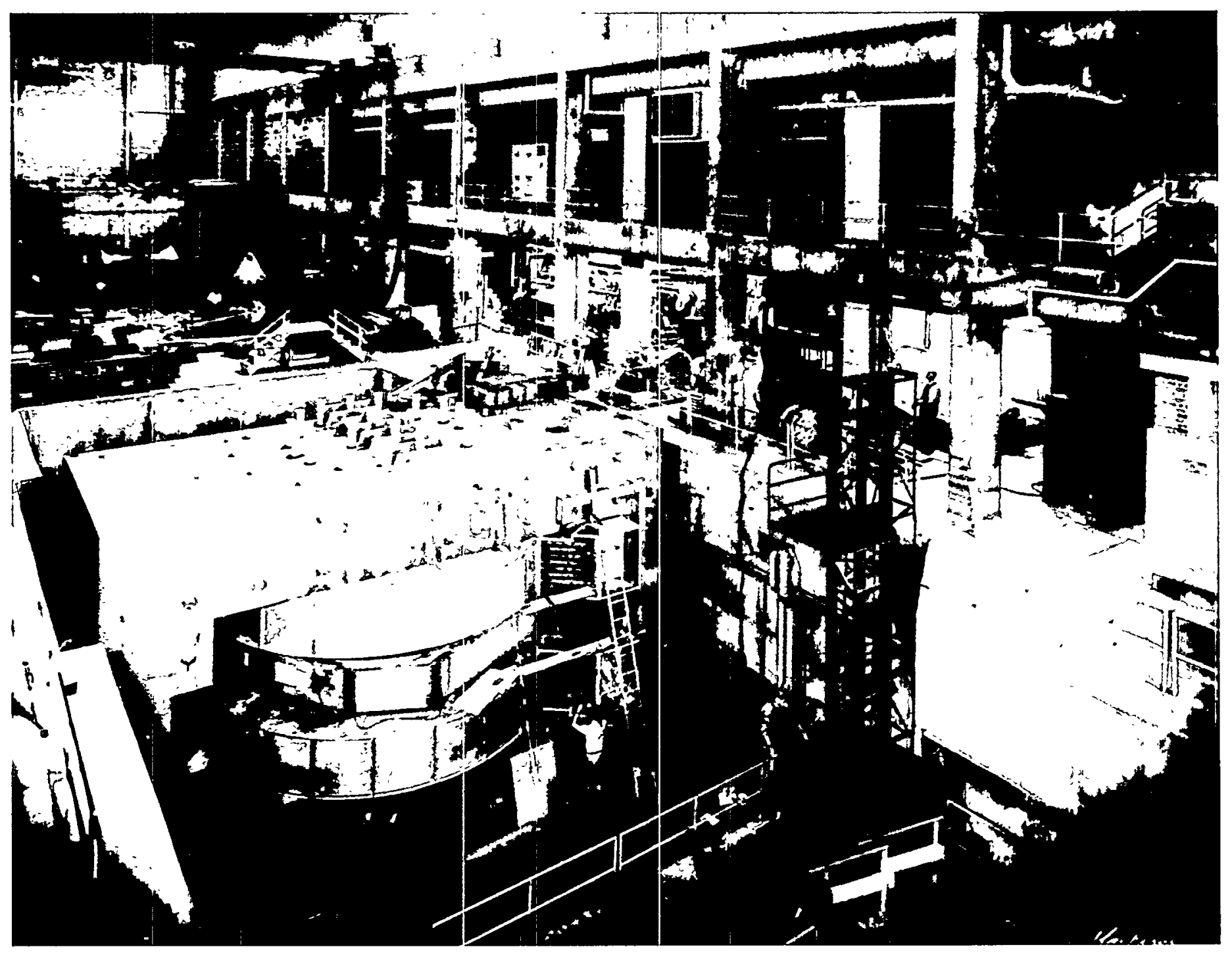

The synchrotron of the University of Chicago as it appeared during construction. A man is standing, center foreground, near the place where beams of pions would emerge and enter the room when pion-scattering experiments were conducted.
Anderson, Fermi, and Marshall are here standing by the cyclotron. Somewhat later in time, the next picture shows Fermi and Andrew Lawson, the Physics Department chairman, entering the Institute building. Three students of Anderson, namely Ron Martin, Morris Glicksman, and Guarang Yodh, are seen in the next photo.

One day soon after I came to Chicago, while we were hunting leaks at the cyclotron, I was sitting in my laboratory reading some reports, when Herbert burst through the door, which, as I have already explained, was often his habit. $\mathrm{He}$ demanded, rather truculently, I thought, to know what I was doing. I said politely that I was thinking about building a liquid hydrogen target to study pion-proton scattering. He said, politely this time, that that was a good idea and that I should get going. So I rose from my chair and went across the street to the West Stands to see
Earl Long, of the Institute for the Study of Metals, who then was very helpful to me in getting the target built rather quickly out of simple materials. The target, featuring sturdiness and speedy construction rather than mechanical elegance, is shown in my laboratory. I am making some changes to the systems.

\section{Experiments on Pion Scattering}

$\mathrm{W}$ e, namely, Anderson, Fermi, and I, then began to measure the total cross sections of hydrogen for negative pions of several energies, followed by the cross sections for the pion charge-exchange reaction, followed by the deuterium and hydrogen cross sections for several energies of negative and of positive pions. 

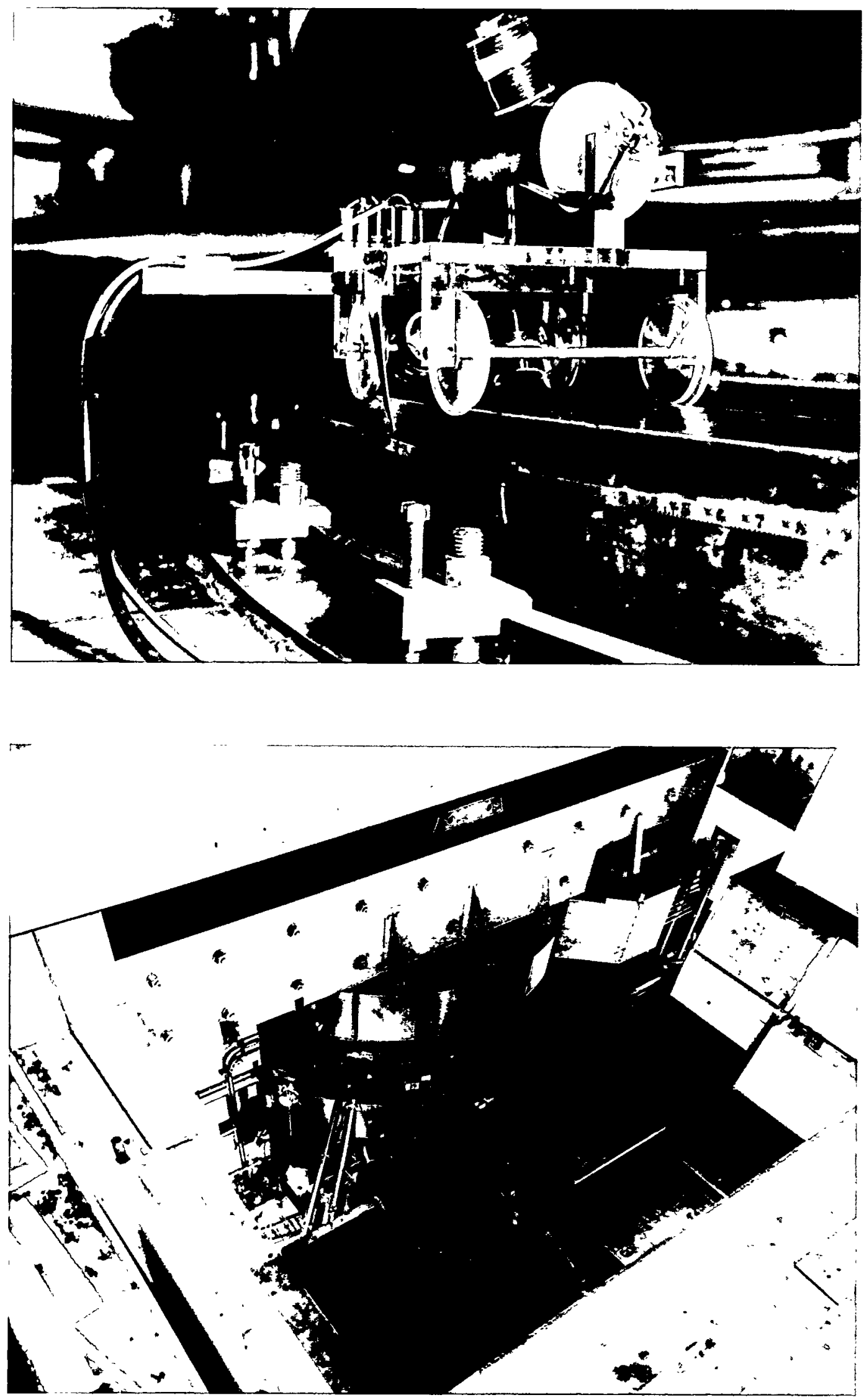

The "trolley car" target, for producing mesons, which ran along the periphery of the cyclotron magnet. The trolley car was designed and personally constructed by E. Fermi.
A view looking down on the Chicago cyclotron through a temporary hole in the shield wall. The square iron shielding plates had slots cut in them through which the meson beams passed from the cyclotron into the room where the experiments were carried out. 
Fermi, Anderson, and Marshall ( $L$ to $R$ ) standing by the meson window of the Chicago cyclotron.
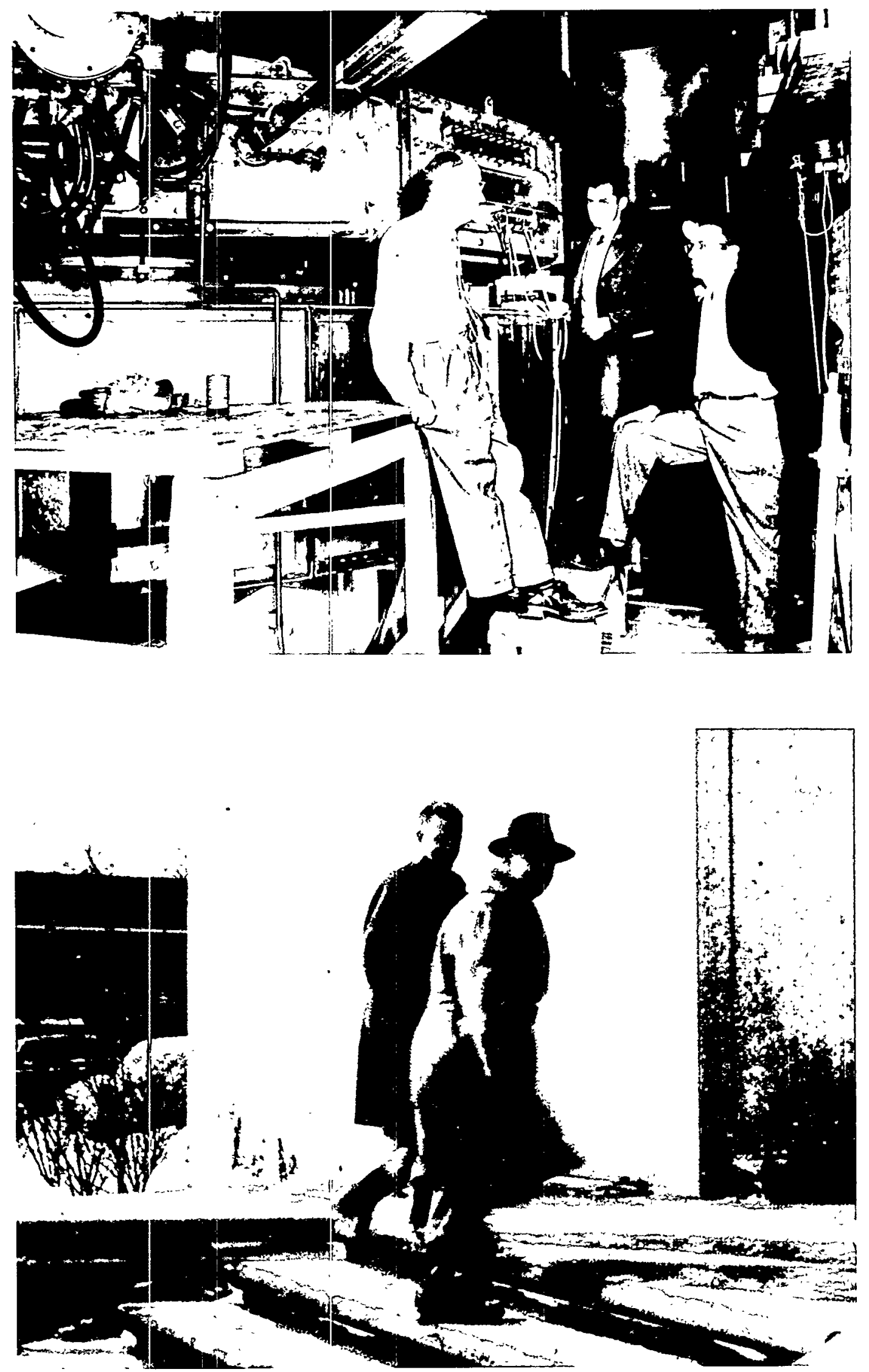

Enrico Fermi (with the hat) and Andrew Lawson entering the Institute building at the University of Chicago. Photo courtesy of $G$. Yodh. 


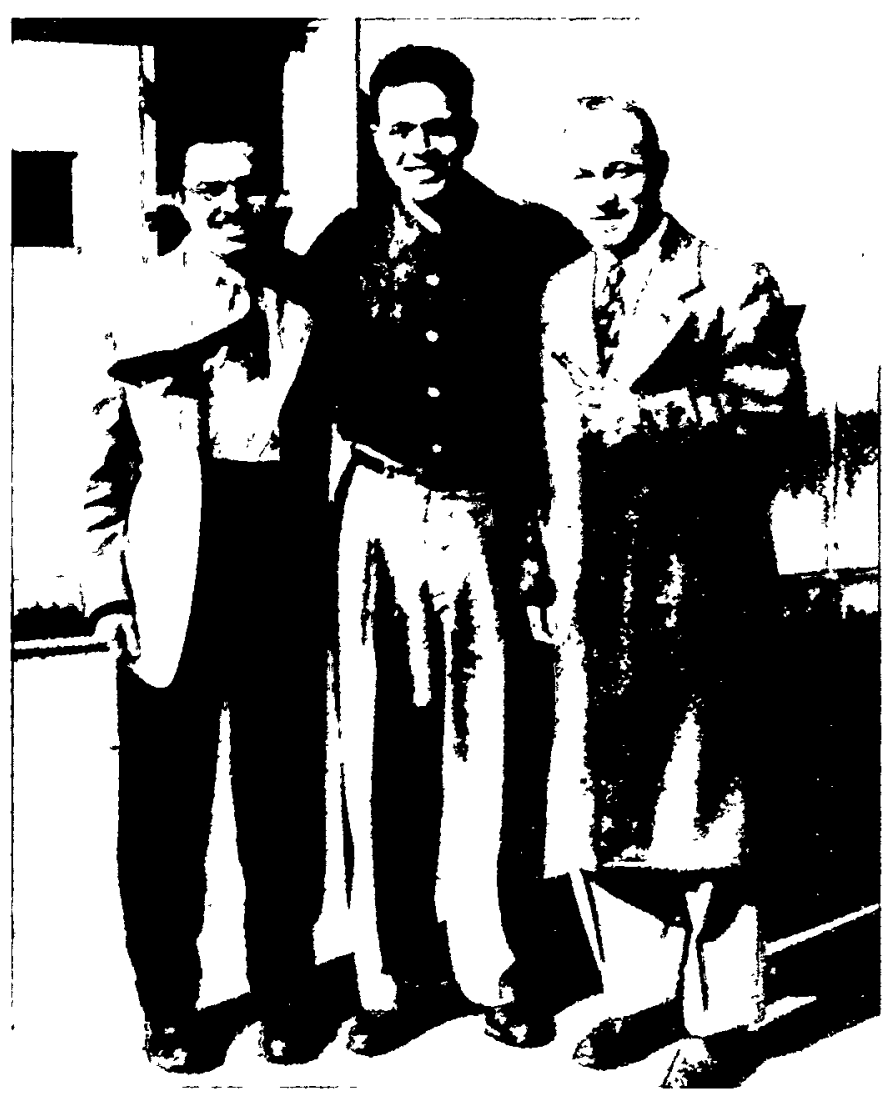

Ron Martin, Morris Glicksman, and Guarang Yodh in front of the Institute's building, about 1955 . Photo courtesy of G. Yodh.

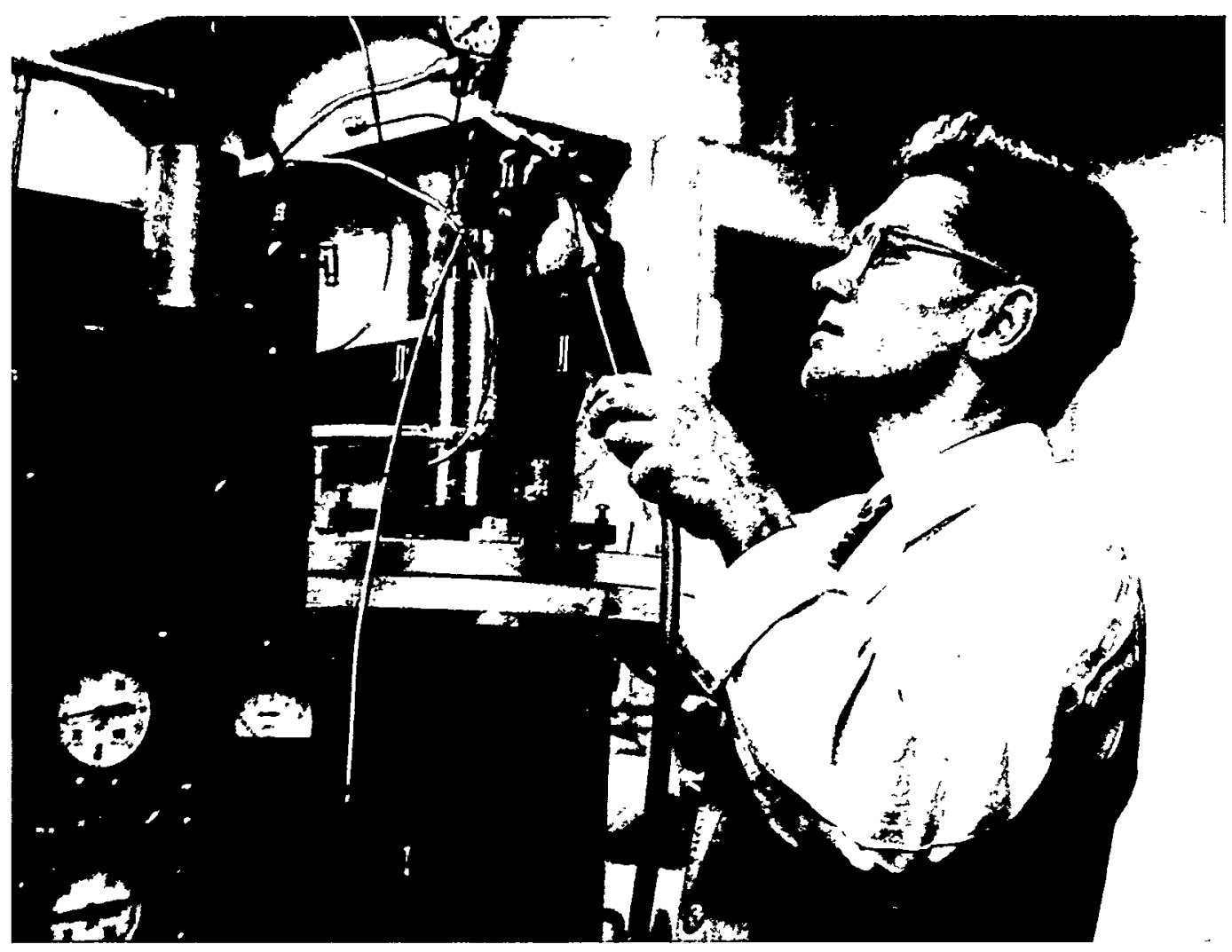

The liquid hydrogen target used in the earliest experiments at Chicago on the scattering of pions from hydrogen, which led to the discovery of the delta. D. Nagle is modifying the target system. 
Later we were joined by Ron Martin and Guarang Yodh. The experiments measured the transmission of the hydrogen; the effective cross section for the reaction in question being simply the negative logarithm of the transmission divided by the number of atoms per square centimeter in the hydrogen target.

The results showed the cross sections for negative pions rising about linearly with the energy up to $100 \mathrm{MeV}$ or so and then appearing to level off at about 60 millibarns, the expected "geometrical" value, i.e., $\pi$ times the pion Compton wavelength squared. This type of energy dependence was expected by the theoretical calculations then in vogue, which used expansions in terms of the pion-nucleon coupling constant ("weak coupling" theory) and assumed the coupling favored P-states (pseudo vector coupling). The positive pion cross sections for a more limited range of energies seemed to be rising in a similar way but at the energies then available had not "leveled off."

\section{Charge Independence and the Resonance}

A t first it puzzled us all: why did the positive pion reactions proceed more strongly than the negative pion reactions, considering that the only channel open for positive pions at low energy was the elastic one,

$$
\pi^{+}+\mathrm{P} \rightarrow \pi^{+}+\mathrm{P},
$$

whereas for negative pions the three reactions,

$$
\begin{aligned}
& \pi^{-}+\mathrm{P} \rightarrow \pi^{-}+\mathrm{p} \quad \text { (elastic scattering), } \\
& \pi^{-}+\mathrm{P} \rightarrow \pi^{0}+\mathrm{N} \quad \text { (charge exchange), and } \\
& \pi^{-}+\mathrm{P} \rightarrow \gamma+\mathrm{N} \quad \text { (radiative capture), }
\end{aligned}
$$

all can proceed.

Anderson gives, in a review article,* an account of how Fermi reacted to a paper by Keith Brueckner, which came, and purported to explain the relative strengths of these several reactions and their energy dependence, and notably predicted a peak in the cross sections (other than the radiative one) at about $180 \mathrm{MeV}$, how quickly Fermi appreciated what Brueckner had to say, and how we regained the initiative.

In his paper Brueckner introduced a phenomenology based on earlier ideas of strong

*H. L. Anderson. Journal De Physique, Supp. au No. 12, Tome 43 (December 1982). pp. c8-101. coupling meson theory and the charge independence of nuclear forces, ideas which had had a long prior history. Forgive me for reminding you of them.

Charge symmetry of the nuclear force:

$$
\begin{aligned}
& \mathrm{NN} \text { force }=\mathrm{P} P \text { force and } \\
& \pi^{-} \mathrm{N} \text { force }=\pi^{+} \mathrm{P} \text { force } .
\end{aligned}
$$

Charge independence:

$\stackrel{N}{\mathrm{~N}}$ ] form a doublet, the nucleon, whose interactions with other nucleons (or pions) is independent of the charge state. The three pions form a triplet, the pion,

$$
\left.\pi: \quad \begin{array}{l}
\pi^{+} \\
\pi^{0} \\
\pi^{-}
\end{array}\right\} .
$$

In analogy with an ordinary spin (J) multiplet, the multiplicity is connected with the isospin; for example, the nucleon isospin is one-half and the corresponding multiplicity is

$$
(2 \times 1 / 2+1)=2 \text {, }
$$

and evidently the pion isospin must be unity since the pion forms the triplet $I_{\pi}=1$. The quantum mechanical rules for combining I-spin states are identical to those for ordinary angular momentum.

The further assumption that there is a resonance in the cross section between pion and nucleon, at about $180-\mathrm{MeV}$ pion energy in the $\mathrm{I}=3 / 2, \mathrm{~J}=3 / 2$ state, led Brueckner to the prediction that near $180 \mathrm{MeV}$ the intensity ratios for the reactions

$$
\begin{aligned}
& \pi^{+}+\mathrm{P} \rightarrow \pi^{+}+\mathrm{P}, \\
& \pi^{-}+\mathrm{P} \rightarrow \pi^{0}+\mathrm{N}, \text { and } \\
& \pi^{-}+\mathrm{P} \rightarrow \pi^{-}+\mathrm{P}
\end{aligned}
$$

would have the relative strength $9: 2: 1$. Some mighty strong indications of this seemed to be in the data although the ratios were not exactly $9: 2: 1$. 


\section{Further Studies:}

Angular Distributions, SU(3), Quarks, and All That...

$\mathrm{O}$ ur Chicago group then proceeded to study the angular dependence as well as the energy dependence of these reactions. Partial wave analysis of the data was done, first using only $S$ and $P$ waves. Later, at Los Alamos, Fermi and Nick Metropolis did the first computer-based analysis, which could require energy continuity of the several partial wave amplitudes. Indeed the $P_{3 / 2}, I=3 / 2$ partial wave amplitude showed a resonant behavior at about $180 \mathrm{MeV}$.

Murray Gell-Mann, then at Chicago, developed the ideas of a deeper underlying symmetry governing the pions and nucleons and the then quite new "strange" particles, the $\Lambda, \Sigma$, and so on, as they came to be known. In Gell-Mann's scheme, the pion-proton "resonance" became the "delta" particle, an I-spin quadruplet, belonging to a decuplet representation of the new symmetry, SU(3). A long and exciting search for new hadrons or strongly interacting particles and resonances was on.

In the sixties, Gell-Mann and George Zweig put forward the idea that underlying the SU(3) symmetry of the strong interactions were three constituent particles, or quarks. Our friend, the delta, posed a puzzle for the scheme, in that the $\Delta^{++}$particle was to be made of three u quarks, all with $S_{z}=+1 / 2, T_{z}=+1 / 2$ in a symmetric space wave function, that is, three identical fermions in a totally symmetric wave function. How could this jibe with the Pauli principle? A new quantum number, color, was invented to solve this problem. The theory of colored quarks and gluons, $Q C D$, has been a success.

\section{The New Generation Accelerator:}

\section{Recent Discoveries}

I 1961, a conference on sector focussed cyclotrons was held at the University of California at Los Angeles. The possibility of $500-$ or $600-\mathrm{MeV}$ proton beams of much higher intensity than those of the synchrocyclotron was discussed. Its applications to nuclear physics were evident. Louis Rosen became convinced that a new-generation accelerator would revitalize nuclear science. He persuaded me, Don Hagerman, Ed Knapp, and others to join in the design and building of the Clinton P. Anderson Meson Physics Facility, called LAMPF. An aerial photo shows the site. The buildings housing the one-half-mile long, 800 $\mathrm{MeV}$ proton linac are visible, and the experimental area is in the foreground. Today, a
Aerial photo of the Clinton P. Anderson Meson Physics Facility, LAMPF.

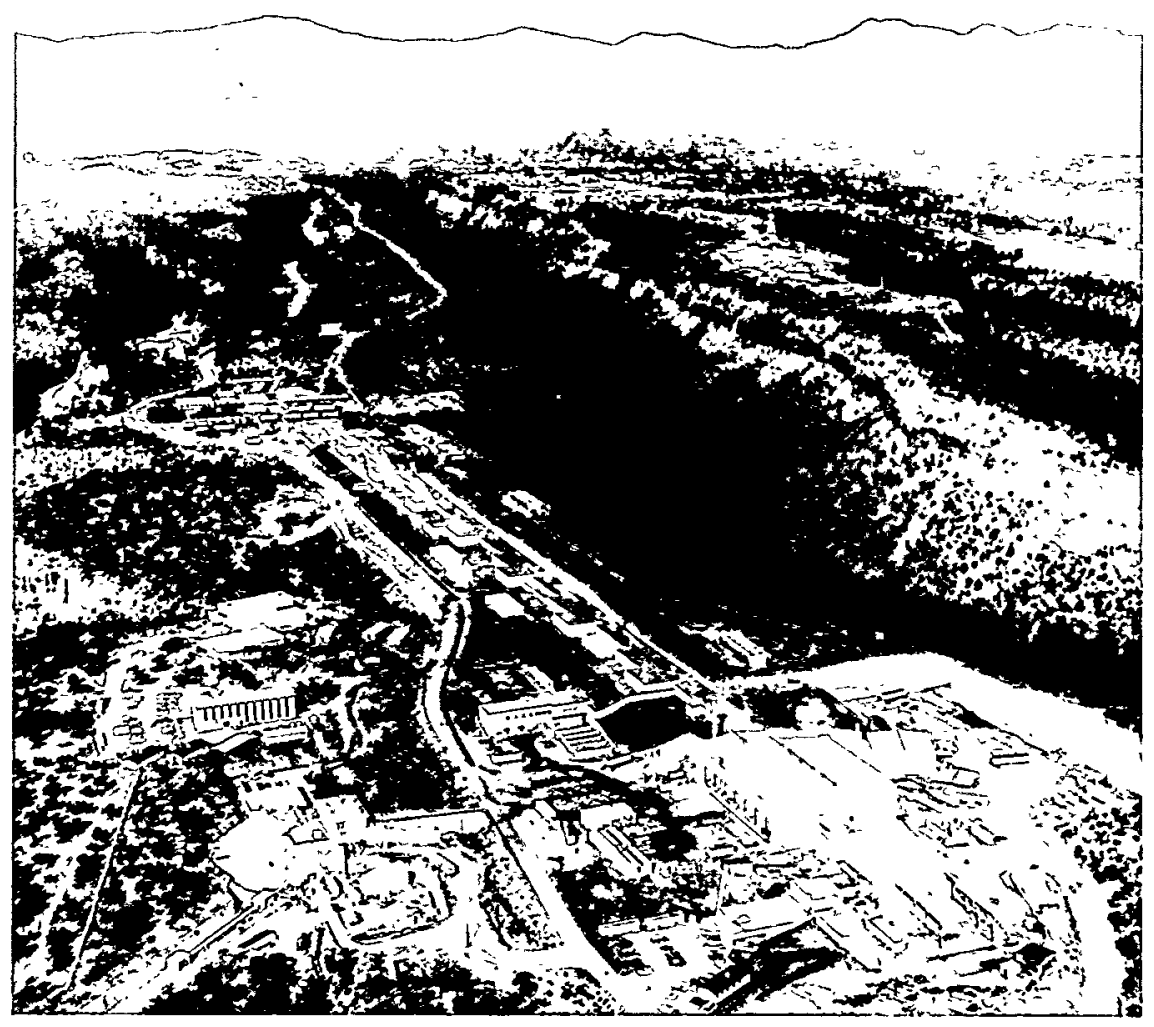


Yields of inelastically scattered pions from ${ }^{13} \mathrm{C}$ as a function of ${ }^{13} \mathrm{C}$ excitation energy. Graph (a) shows the negative pion yield; (b) shows the positive pion yield; (c), the difference of the two yields. Notice that a strong peak at about $9.5 \mathrm{MeV}$ in the negative pion yield is nearly absent in the positive pion yield. The $9.5-\mathrm{MeV}$ line is assigned angular momentum and parity $\left(\frac{9+}{2}\right)$. The ratio of the two intensities turns out to be approximately $9: 1$. decade after its commissioning, some $600 \mathrm{ex}$ periments have been completed. Perhaps 20 per cent of them deal with pion reactions at energies in the resonance region and the properties of the delta, namely its strong characteristic resonance. Other experiments deal with the I-spin and spin characteristics of nuclei. I have selected only a few examples.

The EPICS system at LAMPF consists of a pion channel, a target chamber, and a spectrometer with a resolution of about $150 \mathrm{KeV}$, which makes possible the study of the angular distribution of pions scattered elastically or scattered with excitation of individual levels in the residual nucleus. An interesting nucleus to look at is ${ }^{13} \mathrm{C}$, which, according to the shell model, has a ground state that may be roughly considered as a ${ }^{12} \mathrm{C}$ core plus an extra neutron $\left(1 / 2^{-}\right)$. The measured yield of negative pions and the yields of positive pions are plotted as a function of excitation energy. The data indicate that the ratio is very closely $9: 1$ for a state at $9.5-\mathrm{MeV}$ excitation in ${ }^{13} \mathrm{C}$, just the same ratio we get for a single free neutron, as we discussed earlier. Without giving details here, the overall nuclear physics picture is consistent with this line corresponding to a transition to a state consisting of a $2^{+}$excitation of the ${ }^{12} \mathrm{C}$ core coupled to a $\mathrm{d}_{5 / 2}$ neutron to give overall $9 / 2^{+}$. The literature gives a more careful account, but this seems to be a textbook example of a free neutron-like transition. Like many textbook examples, it is quite rare. Very few levels give $9: 1$ ratios. Most levels in odd-A nuclei require more complicated descriptions, involving strong admixtures of particle-hole configurations. Pion inelastic scattering nevertheless appears to be a very useful tool in sorting out these questions.

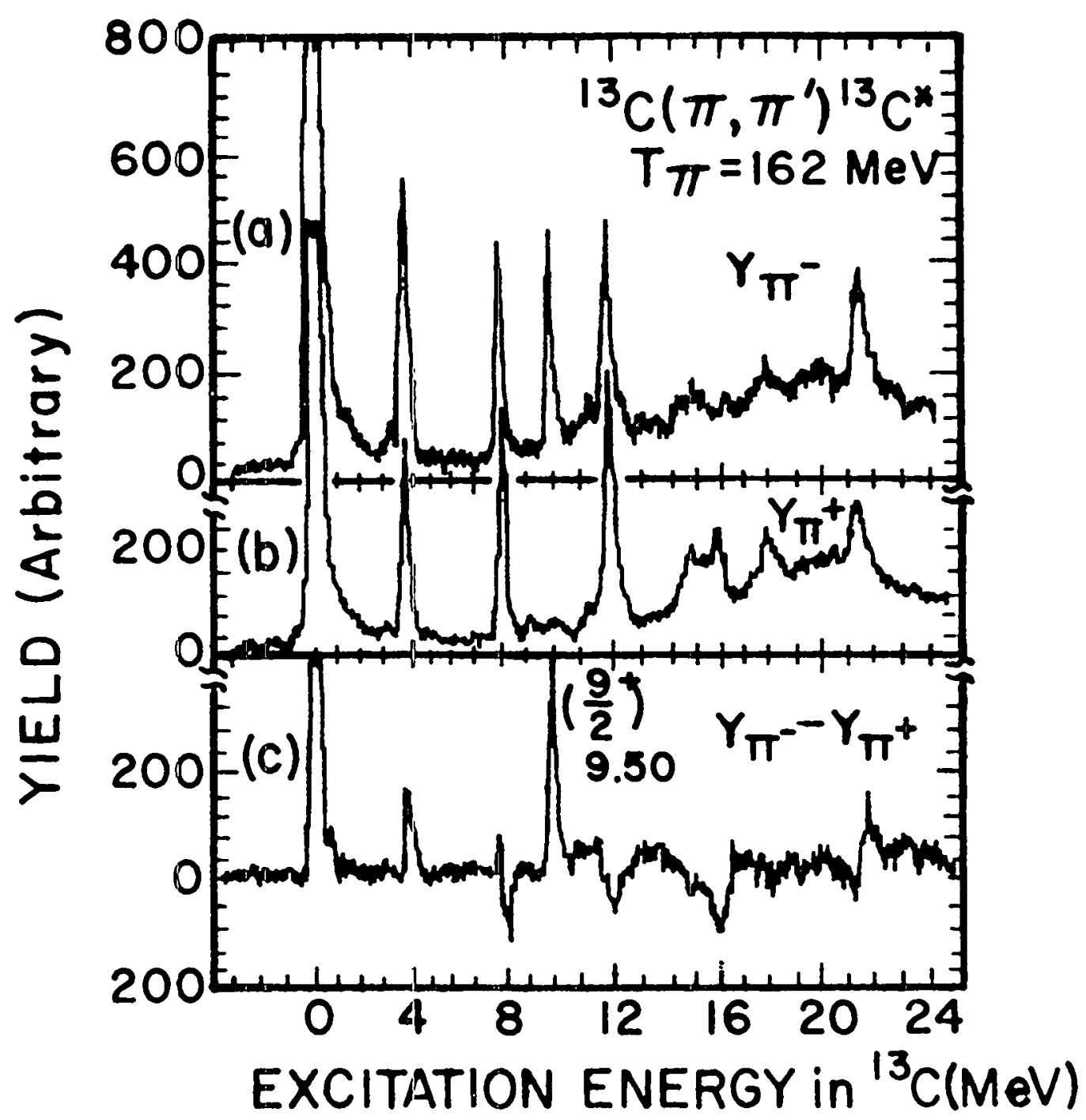




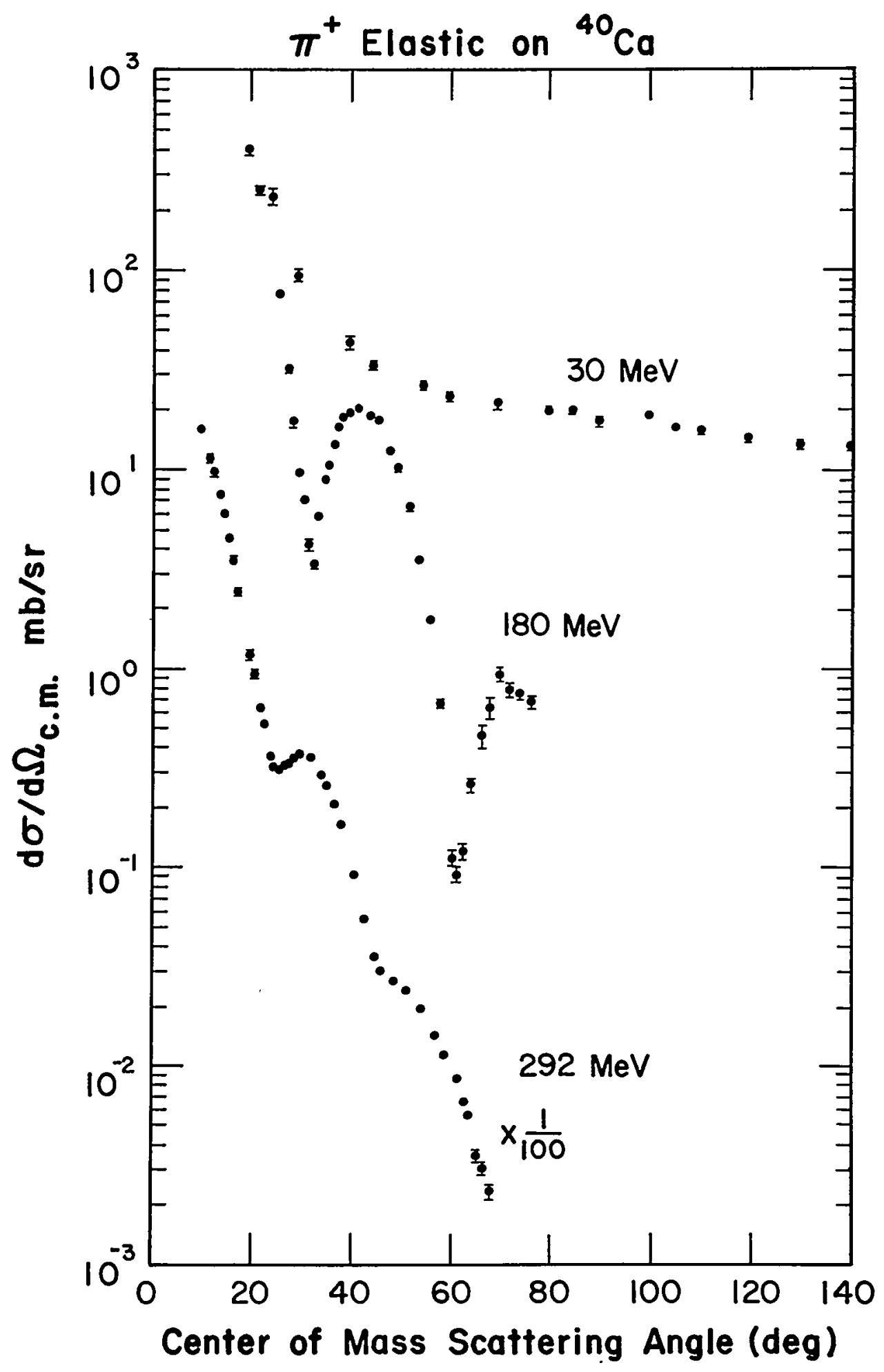

Elastic scattering of $\pi^{+}$from ${ }^{40} \mathrm{Ca}$ for three incident pion energies. At resonance ( $180 \mathrm{MeV}$ ) one sees a pronounced pair of diffraction minima. 
A second example concerns the energy dependence of the angular distributions in the scattering of $\pi^{+}$on ${ }^{40} \mathrm{Ca}$. At the resonance energy, namely $180 \mathrm{MeV}$, the figure shows a classical diffraction pattern for a black disc, namely, $\mathbf{J}_{1}^{2}(\mathrm{kR})$. This implies that the nucleus is absorbing all pions that fall on it, so the nucleus appears black in the light of the pions. Above or below the resonance energy the diffraction minima are filled in, as the nucleus becomes more transparent, expecially for the lighter nuclei.

And now...

Herb's current experiment: parameters of muon decay. $M$. Peters, T. Susuki, M. J. Young, Wayne Kinnison, R. McKee, C. Geisik, and L. Lillberg are shown (L to R).

\begin{abstract}
$\mathrm{A}$ $\mathrm{n}$ interesting question at present is-are there delta-hole components in the wave functions of nuclei? Some evidence supporting this idea has been brought forward, but it is a matter of active discussion how large is the contribution of delta-hole components. Another
\end{abstract}

question is-is there a collective delta-hole giant resonance at about $300 \mathrm{MeV}$ ? Here also evidence exists, but the question is not settled.

Another exciting recent discovery is that of David Bowman and his colleagues, working with the pion charge-exchange reaction on nuclei in the resonance region. They have seen the isovector monopole giant resonance for the first time. The location of the resonance, its width, and the associated angular distribution are about as predicted.

Herb's current experiment at LAMPF (with Wayne Kinnison, Robert McKee, Clifford Hargrove, and John Lillberg) is the study of the beta decay spectrum of the muon. The basic detector is a time projection chamber, and the goal is a tenfold improvement in the accuracy of the Michel parameters that characterize the beta spectrum.

Thirty years later medium energy physics, the delta, and Herb are all vigorous and busy.

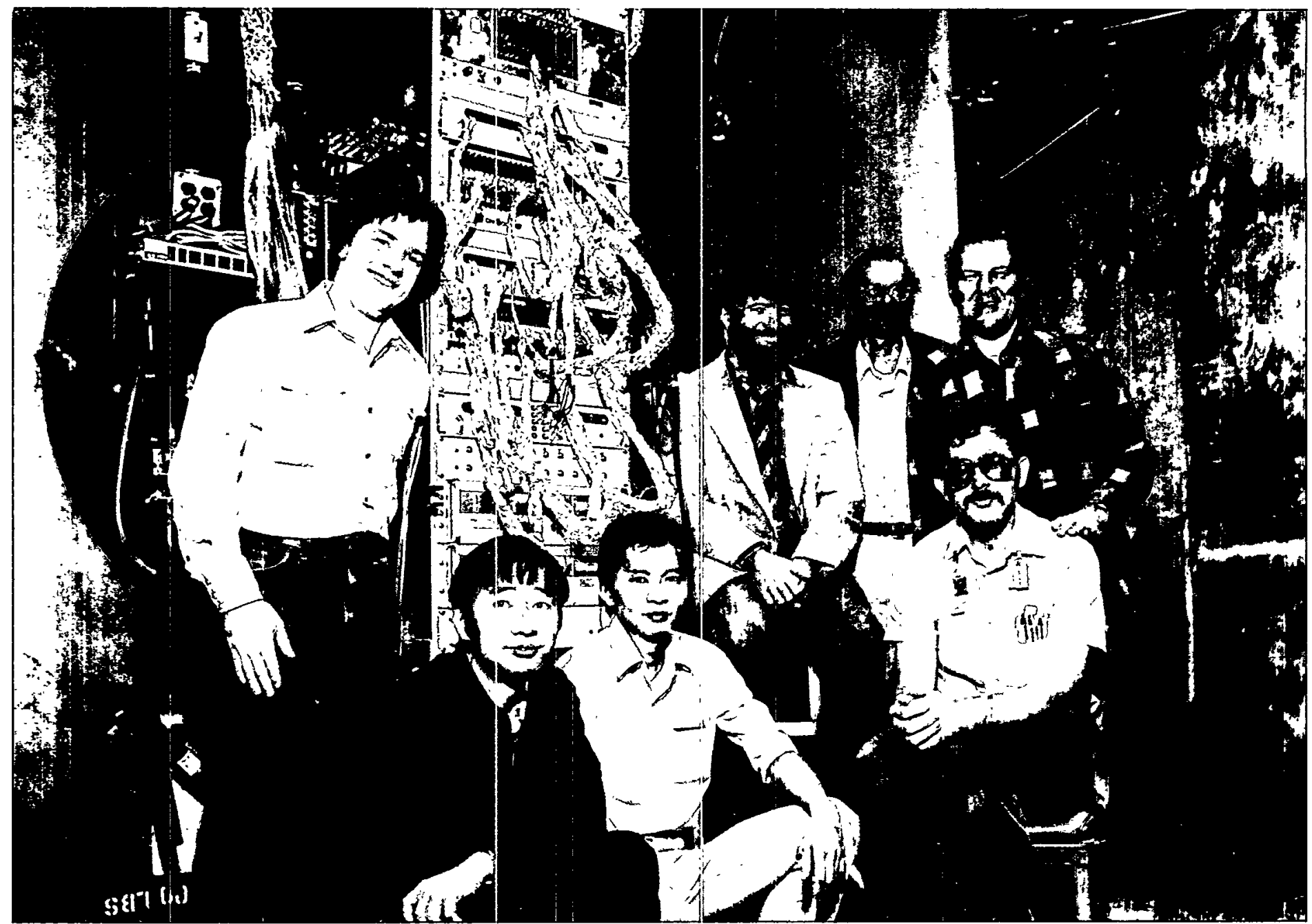

